\title{
Pyramidal Tract Stimulation Restores Normal Corticospinal Tract Connections and Visuomotor Skill after Early Postnatal Motor Cortex Activity Blockade
}

\author{
Iran Salimi, ${ }^{1,2}$ Kathleen M. Friel, ${ }^{1}$ and John H. Martin ${ }^{1,3,4,5}$ \\ ${ }^{1}$ Department of Neuroscience, Columbia University, New York, New York 10032, ${ }^{2}$ Department of Neurosurgery, Mount Sinai Medical School, New York, \\ New York 10029, and Departments of ${ }^{3}$ Psychiatry and ${ }^{4}$ Neurological Surgery, and ${ }^{5}$ The New York State Psychiatric Institute, Columbia University, New \\ York, New York 10032
}

\begin{abstract}
Motor development depends on forming specific connections between the corticospinal tract (CST) and the spinal cord. Blocking CST activity in kittens during the critical period for establishing connections with spinal motor circuits results in permanent impairments in connectivity and function. The changes in connections are consistent with the hypothesis that the inactive tract is less competitive in developing spinal connections than the active tract. In this study, we tested the competition hypothesis by determining whether activating CST axons, after previous silencing during the critical period, abrogated development of aberrant corticospinal connections and motor impairments. In kittens, we inactivated motor cortex by muscimol infusion between postnatal weeks 5 and 7 . Next, we electrically stimulated CST axons in the medullary pyramid $2.5 \mathrm{~h}$ daily, between weeks 7 and 10 . In controls $(n=3)$, CST terminations were densest within the contralateral deeper, premotor, spinal layers. After previous inactivation $(n=3)$, CST terminations were densest within the dorsal, somatic sensory, layers. There were more ipsilateral terminations from the active tract. During visually guided locomotion, there was a movement endpoint impairment. Stimulation after inactivation $(n=6)$ resulted in significantly fewer terminations in the sensory layers and more in the premotor layers, and fewer ipsilateral connections from active cortex. Chronic stimulation reduced the current threshold for evoking contralateral movements by pyramidal stimulation, suggesting strengthening of connections. Importantly, stimulation significantly improved stepping accuracy. These findings show the importance of activity-dependent processes in specifying CST connections. They also provide a strategy for harnessing activity to rescue CST axons at risk of developing aberrant connections after CNS injury.
\end{abstract}

Key words: corticospinal tract; development; locomotion; motor cortex; movement; motion; motor activity; spinal cord

\section{Introduction}

Development of normal motor control depends on forming specific connections between the axons of the corticospinal tract (CST), the key pathway for skilled limb control, and contralateral spinal cord motor circuitry. Achieving this connectivity requires the interplay between guidance cues for target neuron selection (Dottori et al., 1998; Beg et al., 2007) and neural activity for refining connections (Martin, 2005). In kittens, after unilateral inactivation of the motor cortex, the principal origin of the CST in the cat (Ghosh, 1997), the silenced tract is less effective in developing spinal connections than the active tract (Martin, 2005). Silenced CST axon terminations develop a contracted,

\footnotetext{
Received March 12, 2008; revised May 23, 2008; accepted June 10, 2008.

This work was supported by National Institutes of Health Grant NS33835 (J.H.M.) and the United Cerebral Palsy Research and Education Foundation (I.S.). We acknowledge the assistance of Xiu Li Wu for histochemistry and immunohistochemistry, Dan Raz for developing the imaging software, Germa Asfaw and Dr. M. Osman for veterinary care, and R. James Salway, Erin Nunnink, Cedrick Mendoza-Tolentino, Brandon Shulman, and Bernice Sist for help with training and analysis. We thank Jason Carmel for helpful comments on this manuscript.

Correspondence should be addressed to Dr. John H. Martin, Department of Neuroscience, Columbia University, 1051 Riverside Drive, New York, NY 10032. E-mail: jm17@columbia.edu.

DOI:10.1523/JNEUROSCI.1078-08.2008

Copyright $\odot 2008$ Society for Neuroscience $\quad 0270-6474 / 08 / 287426-09 \$ 15.00 / 0$
}

aberrant dorsal distribution in the spinal gray matter (Friel and Martin, 2005). CST axons from the active side, in addition to developing contralateral terminations, develop aberrant ipsilateral spinal terminations on the side normally held by the silenced CST. Remarkably, these changes in CST connectivity in the kitten after unilateral motor cortex inactivation replicate the pattern of aberrant CST connectivity in hemiplegic cerebral palsy (Farmer et al., 1991; Carr et al., 1993). Silencing activity also produces permanent impairments in end-point control during visually guided movements (Martin et al., 2000; Friel et al., 2007).

Our recent finding indicates that there is a protracted period of activity-dependent plasticity in the developing corticospinal (CS) system (Friel and Martin, 2007). Aberrant connections and motor impairments after unilateral inactivation were restored when neural activity in the untreated, active, motor cortex was blocked later in development. We interpreted these findings in the context of our competition hypothesis (Martin, 2005). Blocking the previously active CST reduced the capacity for its spinal terminations to compete for connections with spinal motor circuits. The previously inactive side, facing less competition, could develop proper spinal connections.

In the present study, we conducted a critical direct test of the 
competition hypothesis by determining whether promoting activity-dependent competition by the silenced CS system restores connectivity and function after impaired early CS system development. This experiment also has important clinical significance. Motor impairments in stroke, hemiplegic cerebral palsy, and spinal cord injury are thought to reflect aberrant strengthening or proliferation of undamaged segmental connections that are normally held in check by the CST (Pierrot-Deseilligny and Burke, 2005). We inactivated the motor cortex on one side between postnatal weeks $5-7$, the critical period for establishing CST connections. Then, between 7 and 10 weeks, we electrically stimulated CST axons from the inactivated hemisphere in the medullary pyramidal tract (PT), where CST axons converge. This is when the cortical motor representation is established (Chakrabarty and Martin, 2000) and corticospinal connections strengthen (Meng and Martin, 2003; Meng et al., 2004).

Stimulation after inactivation during the critical period substantially improved CST axon connectivity and movement accuracy, providing direct support for the competition hypothesis. Activity-dependent processes can thus be harnessed to rescue corticospinal tract axons at risk of developing aberrant connections. Our findings also provide a strategy for protecting surviving CST axons from forming aberrant connections and function in human infants at risk of developing hemiplegic cerebral palsy.

\section{Materials and Methods}

Animals were obtained from a supplier accredited by the Association for Assessment and Accreditation of Laboratory Animal Care. Kittens were delivered in litters along with a lactating mother at postnatal day 28. Columbia University and the New York State Psychiatric Institute Institutional Animal Care and Use committees approved all experimental procedures. Three groups of animals were compared: controls (CST terminations, $n=3$; behavior, $n=2$ ), unilateral inactivation (CST terminations, $n=3$; behavior, $n=6$ for impaired and unimpaired sides), and inactivation and stimulation (CST terminations, $n=6$; behavior, $n=4$ ).

We made every effort to make the general behavioral experiences of the three animal groups as similar as possible. All animals were raised in a highly enriched multilevel cage, with constant access to cat toys and facility and laboratory personnel. Inactivation-only and inactivation/ stimulation animals were raised during the entire experimental period, which included the period of stimulation and behavioral testing, with at least one other littermate and the mother. Moreover, our published findings on the effects of unilateral inactivation (Friel and Martin, 2005; Friel et al., 2007) examined animal performance on the ladder task daily, between weeks 7 and 11. Despite this intensive daily enrichment, these animals did not show anatomical or behavioral improvement. Overall, no differences were observed in anatomical or behavioral outcome as a result of subtle differences in day-to-day experiences.

Sensory-motor cortex activity blockade. To block neuronal activity in forelimb primary motor cortex (M1), we infused the $\mathrm{GABA}_{\mathrm{A}}$ agonist muscimol (10 mM in sterile saline; Sigma) using a 28 -gauge hypodermic needle cannula (Durect) (Martin et al., 1999). The cannula was connected with vinyl tubing (Scientific Commodities, size 4) to an osmotic minipump (Alzet, model 2002; Durect) filled with the muscimol solution. Using the metabolic marker cytochrome oxidase, this infusion maximally inhibits a $2.5-3 \mathrm{~mm}$ radius of cortex at the infusion site and has a smaller effect for an additional $4-5 \mathrm{~mm}$ radius (Martin et al., 1999) (i.e., total spread of inactivation, $6.5-8 \mathrm{~mm}$ ). Inactivation reduces immunostaining of parvalbumin, a marker of activity that is also activity dependent (Friel et al., 2007), to a similar extent.

For cannula and pump implantation, animals were administered atropine $(0.04 \mathrm{mg} / \mathrm{kg}$, i.m. $)$ to reduce tracheal secretions. A mixture of acepromazine $(0.03 \mathrm{mg} / \mathrm{kg}$ i.m. ) and ketamine hydrochloride $(32 \mathrm{mg} / \mathrm{kg}$, i.m.) was given to induce anesthesia. The trachea was intubated and the cats were maintained in an areflexive condition during surgery on 1-2\% isoflurane. Animals were given a broad-spectrum antibiotic (cefazolin) at the time of surgery and an analgesic (buprenorphine) afterward.
A 5-mm-wide small craniotomy was made over the forelimb area of M1 (Martin, 1996; Chakrabarty and Martin, 2000). The cannula was inserted $2 \mathrm{~mm}$ below the pial surface and was fixed to the skull with screws and dental acrylic cement. The pump, which was implanted in a subcutaneous pocket between the shoulder blades, delivered muscimol at the rate of $0.5 \mu \mathrm{l} / \mathrm{h}$ during postnatal weeks $5-7$. At the end of the infusion period, the cannula was cut from the pump to terminate infusion. We verified that the animals receiving stimulation after inactivation had the same behavioral impairments during and after cessation of the inactivation. All animals in this study showed abolished or substantially impaired contact placing during the infusion period from weeks 5 to 7 , as in our previous studies (Martin et al., 1999; Friel et al., 2007).

Pyramidal tract electrode and electrode implantation. As in our previous study (Salimi and Martin, 2004), we used an array of Teflon-insulated stainless-steel microwires (50 $\mu$ m diameter; A-M Systems) for stimulation. The microwires (four wires staggered in length by $250 \mu \mathrm{m}$ ) were attached to a stiff tungsten wire (500 $\mu \mathrm{m}$ outer diameter, rounded at the end) using Carbowax (Fisher Scientific) (Palmer, 1978). The Carbowax attachment method permitted detachment of the wires when they were within the brain because it is soluble in a warm aqueous environment; microwires were detached from the carrier above the pia by rinsing the assembly with warm sterile saline.

The microwire electrode array was implanted into the left pyramidal tract (PT) $2 \mathrm{~d}$ after terminating the infusion, and in one animal, one month. Positioning of the electrode array was guided by our previous studies in kittens of the same age (Meng et al., 2004; Salimi and Martin, 2004). The array was inserted stereotactically into the caudal medulla, between 16 and $20^{\circ}$ posterior to anterior angulation. The array was positioned $0.5 \mathrm{~mm}$ lateral and -4 to $-6.00 \mathrm{~mm}$ posterior to interaural zero. The array was driven to the skull floor and then raised $0.5 \mathrm{~mm}$ to allow for the distance between the skull and the pyramid. A silver wire attached to a cranial bone screw was used as the indifferent. The microwires, together with the connector, were fixed in place using cyanoacrylate and dental acrylic cement. We confirmed the position of the electrodes postmortem with the Prussian blue histochemical reaction to stain iron deposits at the stimulation site (supplemental Fig. $1 \mathrm{~A}$, available at www.jneurosci.org as supplemental material) and by locating marking lesions made just before euthanizing the animals on Nissl-stained sections. The day after electrode implantation, we determined the threshold for evoking a motor response from each microwire individually (see below for threshold testing method). We chose one of the microwires as the stimulating electrode based on the lowest threshold for evoking contralateral forelimb movements.

Corticospinal axons tracing. Four weeks before euthanizing each animal, we pressure injected (Picopump; World Precision Instruments) the anterograde tracers biotinylated dextran amine (BDA; Invitrogen; 10\% in PBS) into the forelimb area of M1 of one hemisphere and Lucifer yellow-dextran amine (LY-DA; Invitrogen, $1 \%$ in PBS) into the other. In each M1, we made three tracer injections, $1.5 \mathrm{~mm}$ below the pial surface ( $300 \mathrm{nl}$ each, separated by $1-1.5 \mathrm{~mm}$ ). For determining CST projections from inactivated $\mathrm{M} 1$, tracer injections were made 3-7 d after cessation of the infusion. The three injections were positioned rostral, lateral, and caudal to the muscimol infusion site, as in our previous studies (Friel and Martin, 2007). This ensured that we were examining the previously silenced projection. BDA was used to label the initially inactivated cortex in all but one animal. Injections in the M1 contralateral to the inactivation were made in locations homotopic to the injections in the inactivated M1. Uptake and/or anterograde transport of BDA are not dependent on neural activity. To control for this concern in our previous study and to examine the effects of activity blockade after the critical period, we injected BDA into M1 at week 7, several days before the onset of muscimol infusion (Friel and Martin, 2005). The animal was perfused at week 11 , after 1 month of inactivation and tracer transport. The pattern of CST axon labeling in this animal was identical to the pattern of axonal labeling in age matched controls. Also in that study (Friel and Martin, 2005), we found similar termination patterns regardless of injecting tracers 1 week before infusion (trace from 4 to 7 weeks; inactivated between weeks 5 and 7) or immediately after (inactivated between weeks 5 and 7; trace from 7 to 10 weeks). The pattern of CST axon terminal labeling using LY-DA 
was always the same as with BDA. Finally, effective intracortical transport occurred during local cortical activity blockade by tetrodotoxin (Rhoades et al., 1996).

Stimulation parameters. A constant current stimulator (model 2100; A-M Systems) delivered $45 \mathrm{~ms}$ trains of stimuli $(330 \mathrm{~Hz}, 20 \mu$ s duration biphasic pulses), $2-3 \mathrm{~h}$ per day, once every $3 \mathrm{~s}$, for $21 \mathrm{~d}$ at the end of the inactivation period. Average total stimulation time was $2.6 \mathrm{~h} / \mathrm{d}$. Each day, we used a current that was just sufficient to evoke a contralateral forelimb movement for each stimulus train. To determine the threshold, we started at zero and increased the current in 5-10 $\mu \mathrm{A}$ intervals until a response was observed. Next, we raised the current an additional 20-30 $\mu \mathrm{A}$ and then reduced the current in small steps and noted the value when movements were no longer evoked. The threshold was the average of the currents just evoking a movement during the ascending and descending series. The animals were typically playful while determining the threshold, sometimes making determination difficult. To ensure that we did not underestimate threshold during this period (e.g., the animal's voluntary response was interpreted as a stimulus-evoked response), and risk stimulating below threshold, we sometimes raised the current 5-10 $\mu \mathrm{A}$. Once the current value was established, the animal was placed in an enclosure for the stimulation period and checked every 10-15 min. Animals usually slept for the duration of the stimulation period.

Tissue preparation and staining. At the end of the survival period, animals were deeply anesthetized with sodium pentobarbital $(30 \mathrm{mg} / \mathrm{kg}$, i.v.), injected with heparin (500 units, i.v.), and perfused transcardially with warm saline $\left(39^{\circ} \mathrm{C}\right)$. This was followed by $4 \%$ paraformaldehyde (in $0.1 \mathrm{M} \mathrm{PB}$ ). The brain and spinal cord were removed, postfixed if necessary, and transferred to $20 \%$ sucrose in $0.1 \mathrm{M} \mathrm{PB}$ overnight. Transverse frozen sections $(40 \mu \mathrm{m})$ through the C7-T1 spinal cord were cut. Alternate sections were processed for BDA histochemistry and LY-DA immunocytochemistry. Alternate transverse sections were cut through the medulla for Nissl and Prussian blue staining to identify the location of the electrode array tip and marking lesion. Prussian blue permits visualization of iron deposits at the tip of the electrode, which is the result of the passage of current (supplemental Fig. $1 A$, available at www.jneurosci.org as supplemental material). Sagittal sections were cut through the cortex in both hemispheres to identify the tracer injection sites.

For BDA visualization, sections were incubated in PBS containing 1\% avidin-biotin complex (ABC kit; Vector Laboratories) according to the manufacturers specifications. Sections were incubated with the chromogen diaminobenzidine (DAB; Sigma) for 6-30 min. For visualization of LY-DA, sections were incubated at $4^{\circ}$ overnight in PBS containing $0.01 \%$ rabbit anti-LY antibody (Invitrogen) in blocking buffer (3\% goat serum in $1 \times$ PBS with $0.2 \%$ Tween). After rinsing again, sections were incubated for $2 \mathrm{~h}$ at room temperature in blocking buffer containing $0.2 \%$ anti-rabbit antibody conjugated to peroxidase. After a final rinse, sections were incubated with the chromogen DAB for 5-30 min.

Anatomical data acquisition and analysis. We traced labeled CS axons (terminal and preterminal axons) and marked the locations of axon varicosities, which are putative presynaptic sites. Axon varicosities were defined, as in our previous study, as regions of the axon that are at least three times axonal width ( $\mathrm{Li}$ and Martin, 2002). Care was taken to verify that axon varicosities were not kinks or other irregularities in the axon. Using laser-scanning confocal microscopy, we found that CS axon varicosities colocalize the synaptic vesicle protein synaptophysin (Meng et al., 2004) and appose sites containing puncta of the postsynaptic marker postsynaptic density-95 (D. Trachtenberg and J. Martin, unpublished observations). Transmission electron microscopy confirmed the confocal observations that the CS axon varicosities are filled with synaptic vesicles and appose sites on the postsynaptic membrane with a postsynaptic density (G. Holstein and J. Martin, unpublished observations). Thus, CS axon varicosities, as defined in this study, are likely to mark presynaptic sites, either en passant or terminal. We traced labeled CS axon terminations and varicosities in gray matter of spinal cord using the program Neurolucida (MBF Bioscience). LY-DA- and BDA-labeled axons were traced from transverse spinal sections at $200 \times$ magnification. We also traced the gray matter boundary, Rexed's laminas (see below), and anatomical landmarks. Digital tracings of individual sections were corrected for orientation and aligned with one another according to fiduciary marks (intersection between the gray matter above the central canal and the dorsal median septum). The corrected digital images were exported and measured using custom programs written in Matlab (Mathworks).

To determine local changes in the amount of labeling, digital files of gray matter labeling on individual sections were divided into $52 \times 52 \mu \mathrm{m}$ square regions of interest (ROIs). For each ROI, we computed the mean density of traced axons and marked varicosities. A matrix of mean axon or varicosity density was generated in Matlab that preserved the mediolateral and dorsoventral dimensions of the distribution of label in the gray matter. Using this matrix, we generated topographic maps of the regional distribution of axonal label and varicosities, where local density is represented according to a color scale, from the lowest density (blue) to the highest (red). To compare the distribution of labeling across animals, we used a commercially available program (Morpheus) for the Macintosh computer (Apple) to "warp" axon distributions of each individual animal into a standardized spinal gray matter shape. We then averaged individual animal data from each group. Warping (sometimes termed morphing) systematically stretches the border of the starting image (i.e., from a particular animal) to match that of the final (i.e., average). In doing so, the internal spatial structure of the data are also stretched. This is a standard technique for minimizing spatial variability in human brain imaging, and we have used this technique previously for a similar purpose (Campos et al., 2008). For this study, we use warping as a heuristic, rather than analytic, tool to allow the reader to better appreciate differences in axonal distribution between the animal groups in relation to the laminar organization of the spinal cord. Supplemental Figure S2 (available at www.jneurosci.org as supplemental material) shows the original gray matter outlines for the warping; the final (i.e., warped) outline is shown in Figures $1 C$ and $3 C$. To quantify changes in the distribution of label across animals we determined the amount of label within Rexed's laminas. The following laminas were identified, using Rexed's original criteria (Rexed, 1954) on Nissl- or counter-stained spinal cord sections: laminas 1-3 contain uniformly small cells; lamina 4, a mixture of cell sizes including very large cells not present dorsally, and the ventral border corresponded to a horizontal line from the top of the neck of the dorsal horn; lamina 5, reticulated zone with reduced cell density; lamina 6 anatomically corresponds to the base of the dorsal horn (lamina 6 is more commonly included within the intermediate zone) (see Results); lamina 7 , all ventral territories, exclusive of the medial and lateral motor pools (we distinguished a dorsal region, which corresponded to the portion dorsal to the motor pools, from a ventral region that separated the medial and lateral motor pools); laminas 8 and 9, medial and lateral motor pools, identified by the presence of large cell bodies, which presumably are motoneurons. Local axon and varicosity density, as a proportion of labeling within each of the laminas, was determined using scripts written in Matlab and Microsoft Excel.

Behavioral training and testing. All animals were exposed to the laboratory environment several times a week, beginning between weeks 6 and 7. This enabled obtaining snapshots of performance during the stimulation period and ensured that animals were acclimatized to the environment when testing was done after cessation of stimulation. We examined animals performing a horizontal ladder task (Friel et al., 2007). The ladder was made of Plexiglas ( $88 \mathrm{~cm}$ long, $18.4 \mathrm{~cm}$ wide; $9 \mathrm{~mm}$ square wide rungs placed every $5.8 \mathrm{~cm}$ ), with stationary platforms at either end. Cats were placed at one end of the ladder, and meat cubes were placed at the other end. During testing, the cat walked across the ladder from the start platform to the food reward. To prevent cats from memorizing rung position, we placed them at different positions on the platform for each trial while keeping the distance between rungs constant. This resulted in their starting to step on the rungs with either forelimb. Moreover, the first ladder rung to be stepped on differed from trial to trial. Animals were first introduced to the task during daily sessions of $\sim 10-15 \mathrm{~min}$ and, once acclimated to the lab and the testing apparatus, most animals were tested twice weekly.

Analysis of behavioral performance. Videotapes of testing sessions were imported into a video editing program (iMovie, for the Apple Macintosh computer) and analyzed for forelimb kinematics to measure endpoint control. We used the same analysis criteria as in our previous studies 
(Friel et al., 2007). The forelimb contralateral to inactivation/stimulation is termed the affected limb, and the other, the unaffected limb. We showed that performance of the unaffected limb was identical to that of animals that did not receive muscimol infusion (i.e., untreated control) or received only infusion of the saline vehicle) (Friel et al., 2007). We measured the distance that the tip of the cat's forepaw extended beyond the edge of the rung of the ladder (termed forward distance). We used the program ImageJ, in combination with the program Afloat (Window management tricks for Mac OS X; Microsoft Visual Studio) on the Apple Macintosh computer to measure forward distance. Distance measures from the computer screen were converted to centimeters by scaling according to a calibrated distance on each video file. Trials were not scored if the movement was halted during the step, the movement changed direction, or the animal jumped. Images from the video files were analyzed at $30 \mathrm{~Hz}$. Performance of the inactivation/stimulation animals was compared with data from animals receiving inactivation only, which were reanalyzed from previous studies (Friel and Martin, 2007; Friel et al., 2007).

Statistical analyses. We used the program Statview for the Apple Macintosh computer to determine the statistical significance of differences. Standard and repeated-measures ANOVA was used to compare the dorsoventral distributions of labeling in the inactivation-only and inactivation/stimulation groups. Post hoc testing was used to determine whether particular levels of the spinal cord, known to be relevant to control by the corticospinal terminations, showed significant differences. Pairwise $t$ tests were also used, with correction for multiple comparisons. Linear regression was used to assess the significance of changes in threshold currents with stimulation. Unpaired $t$ tests were used to compare forelimb movement endpoint errors for the inactivated and inactivated/ stimulation groups.

\section{Results}

\section{PT stimulation redistributes aberrant CS terminations and} varicosities ventrally

We examined the regional distribution of labeled CS axon terminals and varicosities, which correspond to putative synapses (Meng et al., 2004), in the C8 spinal segment in each of three animal groups: controls $(n=3)$, unilateral inactivation alone $(n=3)$, unilateral inactivation followed by PT electrical stimulation $(n=6)$. Contralateral CS axon terminals and varicosities are normally densest in the motor territories of the contralateral gray matter, the deepest layer of the dorsal horn (layer 5), and the intermediate zone (layers 6,7), as shown in a representative control (Fig. 1A1,B1) (Friel and Martin, 2005, 2007). This laminar termination pattern is significant because layers 5-7 have been shown to contain premotor interneurons that are active during limb movements in cats (Alstermark and Kümmel, 1990).

Unilateral inactivation between weeks 5 and 7 results in a redistribution of CS terminals and varicosities from the contralateral motor regions in the deep dorsal horn and intermediate zone to more dorsal, mechanosensory laminas (Fig. 1 A2,B2), as reported previously (Friel and Martin, 2005). Stimulation of CS axons in the PT from weeks 7 to 10 , after earlier M1 inactivation, shifted the distribution of axons and varicosities ventrally to the premotor layers (Fig. 1A3,B3). We showed previously that stimulation of sites adjacent to the PT has no effect on CST axon terminal development (Salimi and Martin, 2004). To assess changes across the population of animals examined, the regional axon varicosity map from each animal was warped into a standardized cervical spinal gray matter outline. Averaged composite maps are shown in Figure 1C. Stimulation shifted the focus of maximal axon varicosity density ventrally to the premotor layers (Fig. 1C3), from the dorsal (Fig. 1C2), mechanosensory layers. This shows that stimulation of the developmentally impaired CS system partially reestablished connectivity to the premotor laminas of the cord.
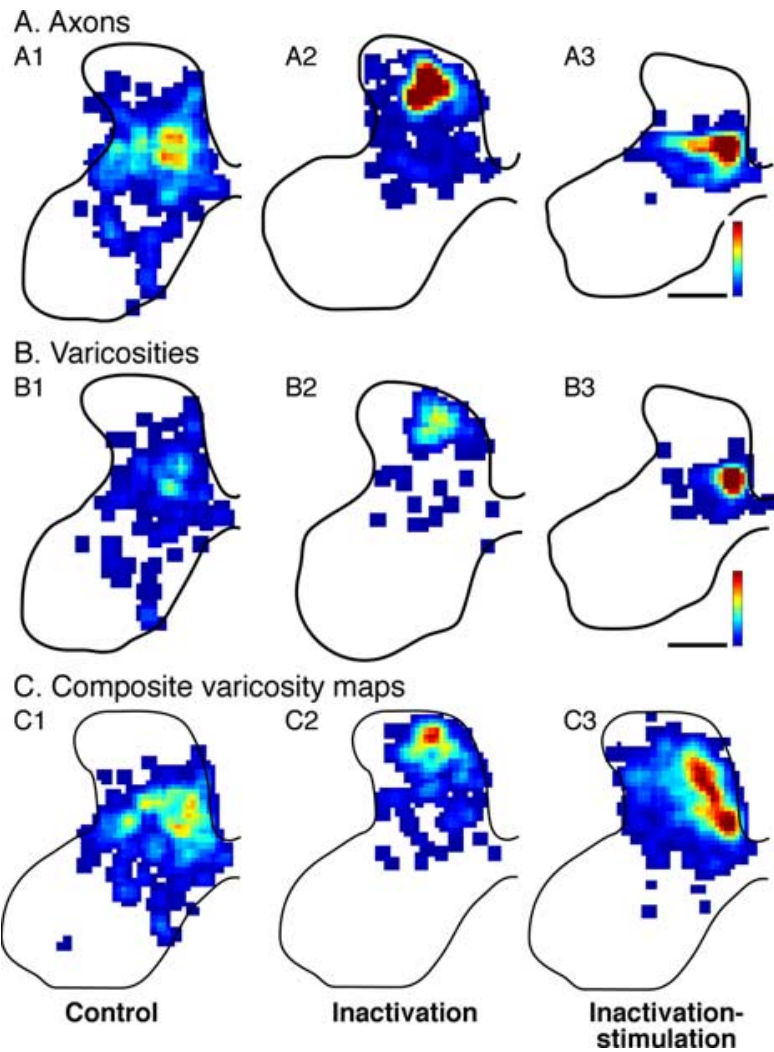

Figure 1. Stimulation after previous $M 1$ inactivation redistributes $C S$ terminations to intermediate and ventral motor laminas. $\boldsymbol{A}, \boldsymbol{B}$, Color-coded topographic maps show the local density of axons $(\boldsymbol{A})$ and varicosities $(\boldsymbol{B})$ in representative animals. $\boldsymbol{C}$, Warped and composite (average; controls, $n=3$; inactivation, $n=3$; inactivation/stimulation, $n=6$ ) topographic maps. $\boldsymbol{A 1}$, B1, C1, Age-matched controls. A2, B2, C2, Inactivated only. A3, B3, C3, Three weeks of stimulation after 2 weeks of inactivation. Red represents the densest area for both axon terminals and varicosities. Color scale: $A, 0-216 \mu \mathrm{m}$ axon/ $\mu \mathrm{m}^{2}$ area; $\boldsymbol{B}, \boldsymbol{C}, 0-8 \times 10^{-2}$ varicosities/ $\mu \mathrm{m}$. Scale bars, $500 \mu \mathrm{m}$.

We quantified the redistribution of CS projections across all animals by plotting the distributions of axon terminations and varicosities in relation to Rexed's laminas (Fig. 2). The control distribution (solid line curve) peaked in laminas 5-7 (dorsal, the deep dorsal horn and upper intermediate zone), whereas the distributions of terminations and varicosities after inactivation alone (Fig. $2 A, B$, light gray bars) peaked in the laminas 1-3 (superficial and middle layers of the dorsal horn), in accordance with the warped image data (Fig. 1C1,2). Importantly, after 3 weeks of daily stimulation after inactivation, the distribution of CS axon terminations and varicosities returned to a pattern similar to controls, with a peak in the more ventral laminas (laminas 4-6) (Fig. 2A, $B$, dark gray bars). Importantly, the distributions of CS axon terminations and varicosities for the inactivated-only and inactivation/stimulation animals were significantly different (ANOVA; axon terminations, $F=3.589, p=0.0052$; varicosities, $F=4.333, p=0.0015)$. In contrast, the distribution of axon terminations and varicosities in control and inactivation/stimulation animals were not different (ANOVA; axon terminations, $F=1.693, p=0.143$; varicosities, $F=1.58, p=0.174)$. Thus, stimulation significantly redistributed the previously silenced CST terminations to be more like that of control animals.

A characteristic feature of the control distribution is that axon terminations and varicosities were significantly denser in laminas 5 and 6 than in laminas 1-3 (axons: $F=9.353, p<0.0002$; Fisher's PLSD, laminas $1-3$ vs lamina $5, p=0.0007$; lamina $6, p<$ 


\section{A. Contralateral axon density}

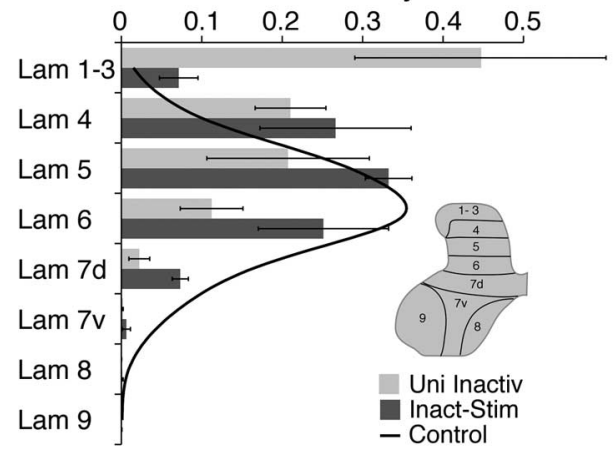

B. Contralateral varicosity density

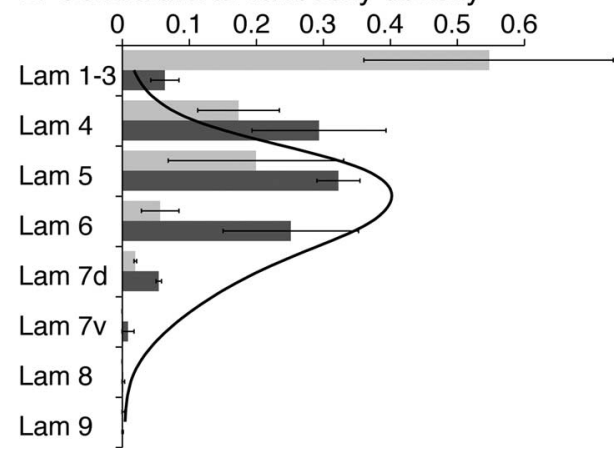

Figure 2. Stimulation after inactivation shifts the distribution of $\mathrm{CS}$ axon termination and varicosities ventrally. $\boldsymbol{A}, \boldsymbol{B}$, Mean ( $\pm \mathrm{SE}$ ) Rexed's laminar distributions of axon terminations $(\boldsymbol{A})$ and boutons $(\boldsymbol{B})$ are plotted for inactivation-only (light gray; $n=3$ ) and inactivation/stimulation (dark gray; $n=6$ ) cats. The lines plot data from control animals $(n=3)$. The inset shows the approximate laminar boundaries.

0.0001; varicosities: $F=6.22, p=0.0019$; Fisher's PLSD, lamina $5, p=0.0012$; lamina $6, p=0.0011)$. In contrast, unilateral inactivation resulted in significantly fewer terminations and varicosities in laminas 5 and 6 than in laminas 1-3 (axons: $F=4.53$, $p=0.0078$; Fisher's PLSD, laminas $1-3$ vs lamina $5, p=0.038$; lamina $6, p=0.0065$; varicosities: $F=4.389, p=0.009$; Fisher's PLSD, lamina 5, $p=0.016$; lamina $6, p=0.0017)$. Finally, like the controls, animals receiving inactivation/stimulation had significantly more terminations and varicosities in laminas 5 and 6 than in laminas $1-3$ (axons: $F=7.624, p<0.0001$; Fisher's PLSD, laminas $1-3$ vs lamina $5, p=0.0012$; lamina $6, p=0.0168$; varicosities: $F=6.286, p=0.0005$; Fisher's PLSD, lamina $5, p=$ 0.0035 ; lamina $6, p=0.026$ ). Our findings indicate that CS system stimulation between weeks 7 and 10 redistributes aberrant contralateral corticospinal connections that occurs after inactivation toward a more normal laminar pattern, with significantly fewer terminations dorsally and more terminations ventrally within territories containing premotor interneurons (Alstermark and Kümmel, 1990).

\section{PT stimulation reduces ipsilateral CST varicosities from the active M1}

Early in development, cats have ipsilateral CS axon terminations that are mostly eliminated by week 7 , with the remaining sparse terminations located ventromedially. The CS tract in humans may undergo a similar refinement (Eyre et al., 2001). After unilateral M1 inactivation, the M1 on the other side (which remained active between weeks 5 and 7; termed the active side) retains its ipsilateral CST terminations (Martin et al., 1999; Friel and Martin, 2007). Figure 3, $A$ and $B$ shows representative exam-
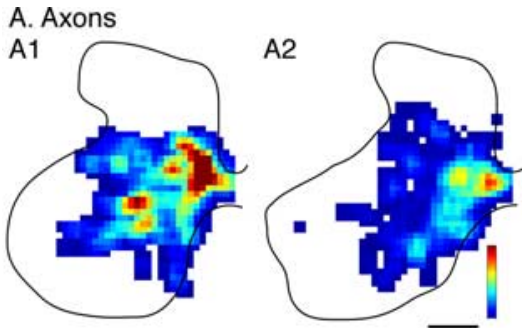

B. Varicosities
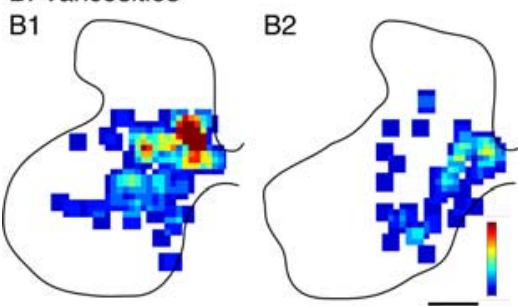

C. Composite varicosity map

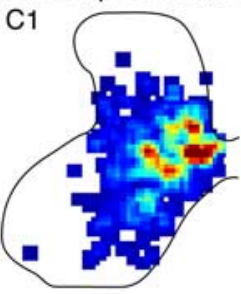

Inactivation

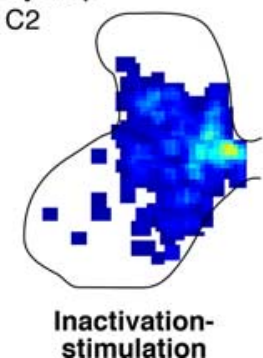

Figure 3. Stimulation after inactivation reduces the density of ipsilateral corticospinal boutons but not axons. The format is similar to Figure 1; representative animals are shown in $\boldsymbol{A}$ and $\boldsymbol{B}$, and a composite average in $\boldsymbol{C}$ (inactivation, $n=3$; inactivation/stimulation, $n=6$; warped data). $\boldsymbol{A} 1, \boldsymbol{B} 1, \mathbf{C 1}, \mathrm{M} 1$ inactivation only. $\boldsymbol{A 2}, \boldsymbol{B} \mathbf{2}, \mathbf{C}$, Inactivation/stimulation. Red represents the densest area for both axon terminals and boutons. Color scale: $\boldsymbol{A}, 0-189 \mu \mathrm{m}$ axon $/ \mu \mathrm{m}^{2}$ area; $\boldsymbol{B}, \boldsymbol{C}, 0-21 \times 10^{-2}$ boutons $/ \mu \mathrm{m}$. Scale bars, $500 \mu \mathrm{m}$.

ples of ipsilateral CS axon terminations and varicosities from the active side (i.e., contralateral to the inactivated M1). In animal receiving inactivation only (Fig. $3 A 1, B 1$ ), the CS projection is present in the ventral dorsal horn and throughout much of the intermediate zone. Maximal density is in the dorsal portion of layer 7. The regional distribution of axon terminations and varicosities is mostly segregated from that of the inactive side, which can be seen by comparing the regions of maximal density in Figures $1, A 2$ and $B 2$, and $3, A 1$ and $B 1$. In the inactivated/stimulated animal, the same laminar axon termination pattern was observed, with the greatest density in the dorsal portion of layer 7 . However, varicosities were consistently less dense (Fig. 3B2). The warped and averaged regional data for the group of animals examined (Fig. 3C) show a substantial reduction in local varicosity density after PT stimulation.

Figure 4 shows the averaged Rexed laminar distributions (laminas 1-9) of ipsilateral CS axon terminations and varicosities. There was a trend toward a reduction in axon terminations in the inactivated/stimulated animals compared with inactivation only (Fig. $4 A$ ), but this was not significant (repeatedmeasures ANOVA; $F=1.963 ; p=0.0834)$. In contrast, the varicosity distribution (Fig. $4 B$ ) in the inactivated/stimulated animals was significantly less than the distribution in the inactivated animals only $(F=2.696 ; p=0.0212)$; the reduction in lamina 7 (dorsal) was significant $(t=2.518 ; p=0.0145)$. This region is just ventral to where stimulation promotes projections from the inactivated side (Fig. 1). Our findings suggest that stim- 


\section{A. Ipsilateral axon density}

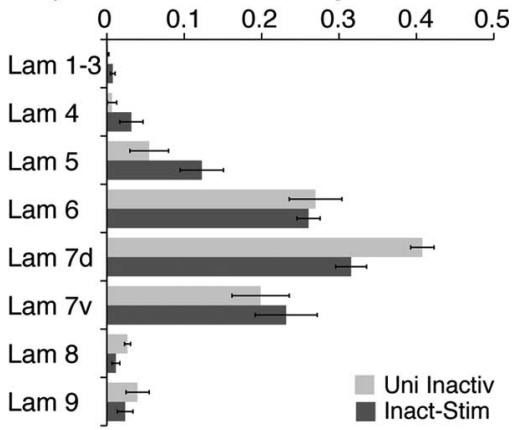

B. Ipsilateral varicosity density

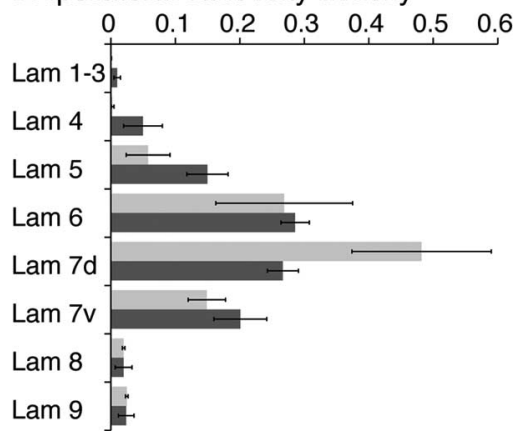

Figure 4. Three weeks of daily stimulation after inactivation reduces the density of ipsilateral varicosities from the nonstimulated CST. The format is the same as in Figure 2. Mean ( $\pm \mathrm{SE}$ ) Rexed's laminar (Lam) distributions of axon terminations $(\boldsymbol{A})$ and boutons $(\boldsymbol{B})$ are plotted for inactivation-only (light gray; $n=3$ ) and inactivation/stimulation (dark gray; $n=6$ ) cats.

ulation produces reciprocal effects on CS varicosities: as the stimulated varicosities of the contralateral CS projection are augmented in the intermediate zone, the nonstimulated varicosities of the ipsilateral CS projection are diminished. It is interesting to note that after unilateral inactivation ipsilateral varicosity density was actually greater than contralateral varicosity density.

\section{Restoration of the regional distribution of CS terminations correlates with progressively reduced motor thresholds during the stimulation period}

We hypothesized that if stimulation strengthens synaptic connections of the previously inactivated CS terminals, we should see a reduction in the threshold for evoking movements by PT stimulation at the end of the stimulation period. We determined the threshold for evoking contralateral responses as part of the daily stimulation protocol to establish the current used (see Materials and Methods). Linear regression analysis, based on the daily current thresholds, revealed significant correlation coefficients in six of the eight cats. The mean threshold on day 1 was $146 \pm 27 \mu \mathrm{A}$, and on day 21 was $88 \pm 16 \mu \mathrm{A}$. The mean slope was $-2.92 \pm 0.79$ $\mu \mathrm{A} / \mathrm{d}$. This threshold reduction is substantially larger than the reduction we observed in the threshold for evoking a CST volley by PT stimulation in anesthetized kittens during the same period of development, which was $-0.35 \mu \mathrm{A} / \mathrm{d}$ (Meng and Martin, 2003). Figure 5 plots the change in current over the stimulation period for each of the eight cats. These findings suggest that the major contribution to the threshold reduction was daily PT stimulation, with a possible smaller age-dependent change, and that this led to a significant increase in the strength of CS connections with spinal motor circuits.

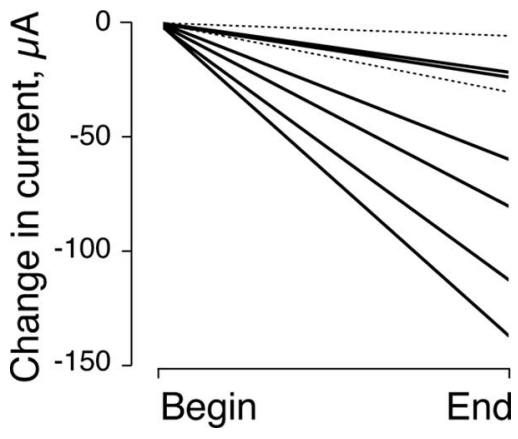

Figure 5. PT stimulation reduces the electrical threshold of previously silenced CST axons for evoking contralateral motor responses. Lines plot the computed regression lines of changes in current threshold for eight cats receiving stimulation after previous inactivation between weeks 8 and 11 . Ordinate plots change in threshold current. Current reduction is indicated by a negative slope. Solid lines are from animals in which there the correlation coefficients were significant (i.e., $p \leq 0.05$ ). Dotted lines plot data from two animals in which the correlation coefficients were not significant.

\section{Stimulation improves visually guided locomotion}

Unilateral M1 inactivation between weeks 5 and 7 abolishes the contract placing reaction during the infusion (Martin et al., 2000; Friel et al., 2007). The placing reaction typically returns within several days after cessation of infusion. However, endpoint errors during reaching and visually guided locomotion persist (Martin et al., 2000; Friel et al., 2007). We next determined whether PT stimulation ameliorated endpoint control errors in visually guided locomotion. We measured changes in the forward distance of the forepaws as animals walked along a horizontal ladder (i.e., distance the front of the paw extended beyond the ladder rung). This is a sensitive and objective measure of the skilled motor impairment produced by M1 inactivation between weeks 5 and 7 . We measured forelimb endpoint at the end of the 3 week stimulation period (week 10). We compared forelimb endpoints in inactivation/stimulation animals $(n=4)$ with findings in animals with inactivation only $(n=6)$, reanalyzed from videotaped data from our previous studies (Friel and Martin, 2007; Friel et al., 2007).

Figure $6 \mathrm{~A}$ compares the forward distance of the affected and unaffected forelimbs in inactivation-only (light gray) and inactivation/stimulation (dark gray) animals. Mean forward distance for the affected limb at the end of the stimulation period was $2.12 \pm 0.11 \mathrm{~cm}(n=4$ cats $)$, which was significantly less than after M1 inactivation alone $(2.72 \pm 0.15 \mathrm{~cm} ; n=6$ cats $)(t=3.185 ; p=$ $0.015)$. Mean forward distance for the unaffected limb was 1.58 $\mathrm{cm} \pm 0.06 \mathrm{~cm}$, which is comparable with our previous studies [1.7 $\pm 0.19 \mathrm{~cm}$ (Friel and Martin, 2007; Friel et al., 2007)]. Thus, animals expressed significantly smaller mean endpoint errors during visually guided locomotion if M1 inactivation was followed by 3 weeks of PT stimulation.

To more directly evaluate improvement of endpoint error produced by stimulation, we computed the difference between the forward distance for the affected and unaffected limbs (i.e., the distance the affected limb overstepped the unaffected limb) for each animal (Fig. 6B). M1 inactivation alone resulted in the affected limb overstepping the unaffected limb by $1.54 \pm 0.12$ $\mathrm{cm}$. In contrast, inactivation followed by CS stimulation resulted in a significantly smaller overstep, only $0.54 \pm 0.058 \mathrm{~cm}$ (unpaired $t$ test; $t=6.264 ; p=0.0002$ ). Thus, $\mathrm{PT}$ stimulation after previous inactivation substantially improved performance of visually guided locomotion and this correlated with substantial restoration of CST connectivity with ventral spinal motor circuits. 

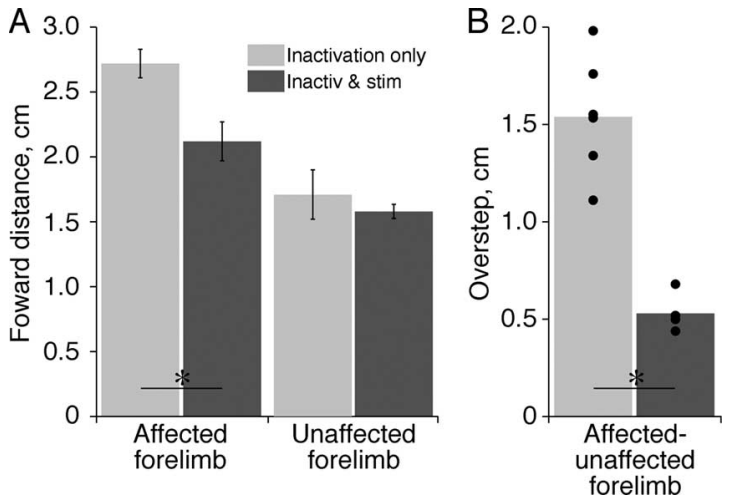

Figure 6. Stimulation after inactivation improves visually guided locomotion by reducing end-point errors of the affected limb. Data presented are from 10-week-old animals. A, Comparison of forward distance in inactivation-only (light gray) and inactivation/stimulation (dark gray) animals. Data for inactivation only were reanalyzed from a large database (Friel and Martin, 2007; Friel et al., 2007). Mean forward distances are as follows: inactivation only, affected limb, $2.72 \pm 0.15 \mathrm{~cm}, n=6$ cats; unaffected limb, $1.7 \pm 0.19 \mathrm{~cm}$; inactivation/stimulation, affected limb, $2.12 \pm 0.11 \mathrm{~cm}$; unaffected limb, $1.58 \pm 0.06 \mathrm{~cm}$. $\boldsymbol{B}$, Comparison of overstepping (difference between mean forward distance for the affected and unaffected limbs for each animal) for M1 inactivation alone (light gray; $n=6$ ) with inactivated/stimulated animals (dark gray; $n=4$ ). Inactivation followed by CS stimulation resulted in a significantly smaller overstep.

\section{Discussion}

Activity-dependent competition can be harnessed to rescue CST axons at risk of developing aberrant, and functionally maladaptive, connections with spinal motor circuits and to improve visually guided movement control. The effects of stimulation of previously silenced CST axons, like alternate inactivation of the initially active motor cortex (Friel and Martin, 2007), achieved a partial restoration of CST connectivity and significantly improved motor function. This similarity can be explained by complementary changes, produced by stimulation and alternate inactivation, in competition between the developing CSTs from each hemisphere for spinal connections. After the initial motor cortex inactivation, CST neurons are at a competitive synaptic "disadvantage" compared with their counterparts from the active hemisphere. Stimulation increased the competitiveness of the disadvantaged CST neurons, whereas silencing of the previously active CS system reduced the synaptic competition faced by the disadvantaged neurons. As discussed below, our finding that activation could boost competition for developing connections with spinal motor circuits has important clinical implications for promoting the function of CST neurons that survive after perinatal injury.

The short daily period of stimulation $(2.5 \mathrm{~h})$ of the impaired CST (i.e., the one inactivated between weeks 5 and 7) produced effects that were nearly as robust as those produced by the continuous period of inactivation ( $24 \mathrm{~h}$ daily) of the unimpaired CST (i.e., the one contralateral to the silenced/impaired CST). Reducing competition by inactivation of the previously active M1 likely exerts its effects through spinal circuits originating from the ventromedial ventral horn, where the aberrant ipsilateral CST axons terminate. This may create a permissive environment that "attracts" outgrowth from the previously silenced CST axons into layers of the spinal cord that contain premotor interneurons (Alstermark and Kümmel, 1990). In contrast, stimulation could confer a heightened intrinsic capacity to overcome the growth suppressive effects of the environment.

The conditions under which activity during development provides instructional signaling or constitutive trophic effects are not understood for the CS system, nor are the roles for activity in refining specific circuit features. For the visual system, electrical stimulation of the optic nerve in dark-reared animals resulted in less-precise orientation tuning in primary visual cortex (Weliky and Katz, 1997). It was proposed that stimulation introduced noise, by synchronous activation of retinal ganglion cell axons in the optic nerve, which disrupted instructional signaling normally provided by spontaneous activity. This raises the question of whether synchronous activation of the CST, which is likely to be uncorrelated with on-going ("intentional") activity, could have led to further development of aberrant or maladaptive connections. However, using both anatomical and functional assays, our findings show that this was not the case; raising the level of activity phasically normalized the topography of CST circuitry in the cord and improved visuomotor control. There are now several examples in which promoting CS system activity leads to specific functional changes, such as chronic motor cortex stimulation augmenting the amplitude of the H-reflex in normal adult rats (Chen et al., 2007) or improving reaching performance after a small motor cortex stroke (Adkins-Muir and Jones, 2003; Adkins et al., 2006). Thus, for the CST it may be sufficient to recruit activity-dependent processes to strengthen CST synapses for achieving adaptive effects. Given our demonstration of the importance of competitive interactions shaping development of connections in the cord, it is tempting to speculate that activityinduced changes in the mature CS system may also be caused by promoting synaptic competition by the stimulated CST axons.

\section{CST circuit changes in hemiplegic cerebral palsy}

Recent findings of Eyre et al. (2007) point to a progressive component to hemiplegic cerebral palsy. Normal infants and those that will develop hemiplegic cerebral palsy have similar transcranial magnetic stimulation (TMS)-evoked motor patterns at 3 months: unilateral TMS evokes bilateral limb motor responses. By 6-12 months, TMS in healthy infants evokes predominantly contralateral effects. In contrast, an aberrant evoked motor pattern emerges in infants that develop hemiplegic cerebral palsy: minimal responses from the damaged cortex and bilateral responses from the undamaged (or less damaged) cortex. We propose that this is explained by an imbalance in activity-dependent competition between the two sides (Martin and Lee, 1999; Martin et al., 1999; Martin, 2005). CST neurons that are initially less competitive in maintaining connections with spinal motor circuits become progressively less competitive as they lose more connections later in development.

CST axons that survive after unilateral perinatal trauma may initially develop sparse and aberrant connections, but have the potential to regain normal connectivity. This potential could be achieved either by enhancing their ability to compete for synaptic space or by reducing synaptic competition that they face from other sources. Direct CST activation, as we have used in this study, or repetitive TMS could be used to activate CST axons to enhance competitiveness. Constraint-induced movement therapy (Charles and Gordon, 2005; Gordon et al., 2006) or deactivating the intact cortex using an inhibitory TMS pulse sequence (Di Lazzaro et al., 2005) might reduce competition. It is important to note that stimulation also promotes CST outgrowth and increases connection strength in adult rats (Brus-Ramer et al., 2007), showing that the effects of activity on CST axons are not restricted to the developing nervous system (Salimi and Martin, 2004). This, combined with the findings of protracted CST development (Koh and Eyre, 1988; Armand et al., 1997; Nezu et al., 
1997; Olivier et al., 1997), suggest that stimulation could rebalance connectivity late in development or in maturity.

\section{CS circuitry and visual guidance of limb control}

Normalization of contralateral connectivity in the cervical spinal cord after stimulation is likely to be key to improving visuomotor control. This is consistent with recovery of motor function after stroke in mature humans, which stresses the importance of the ipsilesional cortex (Ward and Cohen, 2004). We have focused on the contralateral CST projection originating from M1; however, as in humans after M1 lesion, premotor areas may contribute to improved performance in cats after stimulation. Although we have examined the direct segmental CST projection, restitution of other CS system circuits, such as between CST axons and propriospinal systems (Pierrot-Deseilligny and Burke, 2005), could also contribute to improvement. This is because CST axons have collateral branches that project to multiple levels of the spinal cord (Shinoda et al., 1976, 1986; Casale and Light, 1991). It is also theoretically possible that PT stimulation antidromically activated collateral branches of CST axons that project into the brainstem (Keizer and Kuypers, 1984; Ugolini and Kuypers, 1986).

Stimulation also reduced the density of ipsilateral varicosities. Perhaps with a longer period of stimulation, ipsilateral axons would also have been reduced. Normalization of the contralateral terminations (i.e., ventral shift in connections) occurs in association with normalization of ipsilateral terminations (i.e., reduction in connections). It is plausible that the two are causally linked, via reciprocal spinal (Pierrot-Deseilligny and Burke, 2005) or supraspinal circuitry. However, it is unclear whether a reduction in ipsilateral connectivity is beneficial for motor recovery. An adaptive role for the ipsilateral CS system in recovery after unilateral CS system injury in humans is controversial (Ward and Cohen, 2004; Staudt, 2007). In the cat, aberrant ipsilateral CST projections after previous inactivation are adaptive, and their presence improves performance of the affected limb (Martin et al., 2000). In the intact spinal cord, ipsilateral CST axon terminations comprise $10-15 \%$ of the population (Brösamle and Schwab, 1997; Lacroix et al., 2004) and, thus, are likely to be adaptive because of their large number. Ipsilateral control can also be mediated by indirect routes, via brainstem motor paths. Studies by Edgley, Jankowska, and colleagues (Edgley et al., 2004; Jankowska and Edgley, 2006; Jankowska et al., 2006) stress the importance of corticoreticulospinal networks in transmitting control signals to spinal motor circuits bilaterally. Moreover, ipsilateral actions could occur via callosal projections. To a certain degree, movements of one limb routinely depend on control of the other limb. Whether explicit, as during bimanual manipulations, or as a consequence of postural interactions, such as stabilizing an object with one limb and taking action with the other, bilateral control is likely reflected in some degree of control by the ipsilateral CS system.

\section{Combinatorial rehabilitation approaches with hemiplegic cerebral palsy}

Stimulation of the damaged nervous CST likely targets multiple repair mechanisms. Lesion of the CST on one side in mature rats leads to ipsilateral outgrowth of intact CST axons into the territory occupied by the previously lesioned axons, and stronger connections (Brus-Ramer et al., 2007). Stimulation of CST axons in the intact nervous system also leads to outgrowth and, importantly, significantly stronger CST connections than after injury. Combining stimulation and injury resulted in significantly stronger connections than either of the two alone. Thus, stimulation significantly augments lesion-induced plasticity, possibly through separate effects on axonal outgrowth and connection strength. With this in mind, development of therapies for prevention and rehabilitation of motor control impairments in patients with cerebral palsy could combine different mechanisms, each at the level of motor circuitry. CST stimulation could be combined with behavioral training (Butefisch et al., 2004) to target circuits that are concurrently activated by the stimulus and recruited by task performance. Stimulation could be combined with constraint inducted movement therapy of the unaffected side to increase the "activation dosage." Manipulation of activitydependent processes globally, as with constraint or motor performance, or selectively, as with CST stimulation, alone or in combination, could prove fruitful in promoting motor function after perinatal CS system injury.

\section{References}

Adkins DL, Campos P, Quach D, Borromeo M, Schallert K, Jones TA (2006) Epidural cortical stimulation enhances motor function after sensorimotor cortical infarcts in rats. Exp Neurol 200:356-370.

Adkins-Muir DL, Jones TA (2003) Cortical electrical stimulation combined with rehabilitative training: enhanced functional recovery and dendritic plasticity following focal cortical ischemia in rats. Neurol Res 25:780-788.

Alstermark B, Kümmel H (1990) Transneuronal transport of wheat germ agglutinin conjugated horseradish peroxidase into last order spinal interneurones projecting to acromio- and spinodeltoideus motoneurones in the cat. 2. Differential labelling of interneurones depending on movement type. Exp Brain Res 80:96-103.

Armand J, Olivier E, Edgley SA, Lemon RN (1997) Postnatal development of corticospinal projections from motor cortex to the cervical enlargement in the macaque monkey. J Neurosci 17:251-266.

Beg AA, Sommer JE, Martin JH, Scheiffele P (2007) alpha2-Chimaerin is an essential EphA4 effector in the assembly of neuronal locomotor circuits. Neuron 55:768-778.

Brösamle C, Schwab ME (1997) Cells of origin, course, and termination patterns of the ventral, uncrossed component of the mature rat corticospinal tract. J Comp Neurol 386:293-303.

Brus-Ramer M, Carmel JB, Chakrabarty S, Martin JH (2007) Electrical stimulation of spared corticospinal axons augments connections with ipsilateral spinal motor circuits after injury. J Neurosci 27:13793-13801.

Bütefisch CM, Khurana V, Kopylev L, Cohen LG (2004) Enhancing encoding of a motor memory in the primary motor cortex by cortical stimulation. J Neurophysiol 91:2110-2116.

Campos LW, Chakrabarty S, Haque R, Martin JH (2008) Regenerating motor bridge axons refine connections and synapses on lumbar motoneurons to bypass chronic spinal cord injury. J Comp Neurol 506:838-850.

Carr LJ, Harrison LM, Evans AL, Stephens JA (1993) Patterns of central motor reorganization in hemiplegic cerebral palsy. Brain 116:1223-1247.

Casale EJ, Light AR (1991) The terminations of single, physiologically identified, somatosensory, corticospinal tract axons in the lumbar spinal cord of the cat. J Neurophysiol 66:1738-1749.

Chakrabarty S, Martin JH (2000) Postnatal development of the motor representation in primary motor cortex. J Neurophysiol 84:2582-2594.

Charles J, Gordon AM (2005) A critical review of constraint-induced movement therapy and forced use in children with hemiplegia. Neural Plast 12:245-261; discussion 263-272.

Chen XY, Pillai S, Chen Y, Wang Y, Chen L, Carp JS, Wolpaw JR (2007) Spinal and supraspinal effects of long-term stimulation of sensorimotor cortex in rats. J Neurophysiol 98:878-887.

Di Lazzaro V, Pilato F, Saturno E, Oliviero A, Dileone M, Mazzone P, Insola A, Tonali PA, Ranieri F, Huang YZ, Rothwell JC (2005) Theta-burst repetitive transcranial magnetic stimulation suppresses specific excitatory circuits in the human motor cortex. J Physiol 565:945-950.

Dottori M, Hartley L, Galea M, Paxinos G, Polizzotto M, Kilpatrick T, Bartlett PF, Murphy M, Köntgen F, Boyd AW (1998) EphA4 (Sek1) receptor tyrosine kinase is required for the development of the corticospinal tract. Proc Natl Acad Sci U S A 95:13248-13253.

Edgley SA, Jankowska E, Hammar I (2004) Ipsilateral actions of feline corticospinal tract neurons on limb motoneurons. J Neurosci 24:7804-7813. Eyre JA, Taylor JP, Villagra F, Smith M, Miller S (2001) Evidence of activity- 
dependent withdrawal of corticospinal projections during human development. Neurology 57:1543-1554.

Eyre JA, Smith M, Dabydeen L, Clowry GJ, Petacchi E, Battini R, Guzzetta A, Cioni G (2007) Is hemiplegic cerebral palsy equivalent to amblyopia of the corticospinal system? Ann Neurol 62:493-503.

Farmer SF, Harrison LM, Ingram DA, Stephens JA (1991) Plasticity of central motor pathways in children with hemiplegic cerebral palsy. Neurology 41:1505-1510.

Friel KM, Martin JH (2005) Role of sensory-motor cortex activity in postnatal development of corticospinal axon terminals in the cat. J Comp Neurol 485:43-56.

Friel KM, Martin JH (2007) Rebalancing corticospinal activity promotes recovery of motor skill and anatomical integrity after inactivation during a critical period. J Neurosci 27:11083-11090.

Friel KM, Drew T, Martin JH (2007) Differential activity-dependent development of corticospinal control of movement and final limb position during visually-guided locomotion. J Neurophysiol 97:3396-3406.

Ghosh S (1997) Identification of motor areas of the cat cerebral cortex based on studies of cortical stimulation and corticospinal connections. J Comp Neurol 380:191-214.

Gordon AM, Charles J, Wolf SL (2006) Efficacy of constraint-induced movement therapy on involved upper-extremity use in children with hemiplegic cerebral palsy is not age-dependent. Pediatrics 117:e363-e373.

Jankowska E, Edgley SA (2006) How can corticospinal tract neurons contribute to ipsilateral movements? A question with implications for recovery of motor functions. Neuroscientist 12:67-79.

Jankowska E, Stecina K, Cabaj A, Pettersson LG, Edgley SA (2006) Neuronal relays in double-crossed pathways between feline motor cortex and ipsilateral hindlimb motoneurones. J Physiol 575:527-541.

Keizer K, Kuypers HG (1984) Distribution of corticospinal neurons with collaterals to lower brain stem reticular formation in cat. Exp Brain Res 54:107-120.

Koh TH, Eyre JA (1988) Maturation of corticospinal tracts assessed by electromagnetic stimulation of the motor cortex. Arch Dis Child 63:1347-1352.

Lacroix S, Havton LA, McKay H, Yang H, Brant A, Roberts J, Tuszynski MH (2004) Bilateral corticospinal projections arise from each motor cortex in the macaque monkey: a quantitative study. J Comp Neurol 473:147-161.

Li Q, Martin JH (2002) Postnatal development of connectional specificity of corticospinal terminals in the cat. J Comp Neurol 447:57-71.

Martin JH (1996) Differential spinal projections from the forelimb areas of the rostral and caudal subregions of primary motor cortex in the cat. ExpBrain Res 108:191-205.

Martin JH (2005) The corticospinal system: from development to motor control. Neuroscientist 11:161-173.
Martin JH, Lee SJ (1999) Activity-dependent competition between developing corticospinal terminations. Neuroreport 10:2277-2282.

Martin JH, Kably B, Hacking A (1999) Activity-dependent development of cortical axon terminations in the spinal cord and brain stem. Exp Brain Res 125:184-199.

Martin JH, Hacking A, Donarummo L (2000) Impairments in prehension produced by early postnatal sensorimotor cortex activity blockade. J Neurophysiol 83:895-906.

Meng Z, Martin JH (2003) Postnatal development of corticospinal synaptic actions. J Neurophysol 90:683-692.

Meng Z, Li Q, Martin JH (2004) The transition from development to motor control function in the corticospinal system. J Neurosci 24:605-614.

Nezu A, Kimura S, Uehara S, Kobayashi T, Tanaka M, Saito K (1997) Magnetic stimulation of motor cortex in children: maturity of corticospinal pathway and problem of clinical application. Brain Dev 19:176-180.

Olivier E, Edgley SA, Armand J, Lemon RN (1997) An electrophysiological study of the postnatal development of the corticospinal system in the macaque monkey. J Neurosci 17:267-276.

Palmer C (1978) A microwire technique for recording single neurons in unrestrained animals. Brain Res Bull 3:285-289.

Pierrot-Deseilligny E, Burke D (2005) The circuitry of the human spinal cord. Cambridge, UK: Cambridge UP.

Rexed B (1954) A cytoarchitectonic atlas of the spinal cord in the cat. J Comp Neurol 100:297-379.

Rhoades RW, Crissman RS, Bennett-Clarke CA, Killackey HP, Chiaia NL (1996) Development and plasticity of local intracortical projections within the vibrissae representation of the rat primary somatosensory cortex. J Comp Neurol 370:524-535.

Salimi I, Martin JH (2004) Rescuing transient corticospinal terminations and promoting growth with corticospinal stimulation in kittens. J Neurosci 24:4952-4961.

Shinoda Y, Arnold AP, Asanuma H (1976) Spinal branching of corticospinal axons in the cat. Exp Brain Res 26:215-234.

Shinoda Y, Yamaguchi T, Futami T (1986) Multiple axon collaterals of single corticospinal axons in the cat spinal cord. J Neurophysiol 55:425-448.

Staudt M (2007) (Re-)organization of the developing human brain following periventricular white matter lesions. Neurosci Biobehav Rev 31:1150-1156.

Ugolini G, Kuypers HG (1986) Collaterals of corticospinal and pyramidal fibres to the pontine grey demonstrated by a new application of the fluorescent fibre labelling technique. Brain Res 365:211-227.

Ward NS, Cohen LG (2004) Mechanisms underlying recovery of motor function after stroke. Arch Neurol 61:1844-1848.

Weliky M, Katz LC (1997) Disruption of orientation tuning in visual cortex by artificially correlated neuronal activity. Nature 386:680-685. 